\author{
RESEARCH ARTICLE \\ 10.1029/2018JC014638 \\ Key Points: \\ - Estimated spectral properties are \\ signed quantities associated to both \\ wind speed and direction \\ - Distinct sensitivity to wind direction \\ can help to retrieve ocean surface \\ wind vector from SAR measurements \\ - Global mapping and analysis confirm \\ capabilities to enforce wind and wave \\ climate studies
}

Correspondence to:

H. Li,

Huimin.Li@ifremer.fr

Citation:

Li, H., Chapron, B., Mouche, A. A., \& Stopa, J. E. (2019). A new ocean SAR cross-spectral parameter: Definition and directional property using the global Sentinel-1

measurements. Journal of Geophysical Research: Oceans, 124, 1566-1577.

https://doi.org/10.1029/2018JC014638

Received 11 OCT 2018

Accepted 20 FEB 2019

Accepted article online 21 FEB 2019

Published online 7 MAR 2019

\section{A New Ocean SAR Cross-Spectral Parameter: Definition and Directional Property Using the Global Sentinel-1 Measurements}

\author{
Huimin $\mathrm{Li}^{1}{ }^{\mathbb{D}}$, Bertrand Chapron ${ }^{1}$, Alexis Mouche ${ }^{1} \mathbb{D}$, and Justin E. Stopa ${ }^{2} \mathbb{D}$ \\ ${ }^{1}$ Ifremer, University Brest, CNRS, IRD, Laboratoire dOcanographie Physique et Spatiale (LOPS), IUEM, Brest, France, \\ ${ }^{2}$ Department of Ocean and Resources Engineering, University of Hawaii at Manoa, Honolulu, USA
}

\begin{abstract}
Space-borne synthetic aperture radar (SAR) measurements have already been proven to be invaluable in detecting and quantifying properties of ocean swell systems. With the improved spatial resolution, Sentinel-1 wave mode measurements can further be extended toward shorter-scale waves, that is, within the surface wave equilibrium range. In this study, a new parameter is derived from filtering SAR image cross-spectra around range-traveling intermediate wind waves (wavelengths $15-20 \mathrm{~m}$ ). This parameter captures both the radar cross-section variability and its time evolution. Given the dependence of intermediate waves on local wind field, this parameter is statistically documented to confirm its sensitivity to both wind speed and wind direction. Comparable to Doppler estimate, the signed parameter can be used to reduce the wind direction ambiguity in the inversion of high-resolution wind fields from SAR imagery. In addition, under complex environmental situations over a polar low event, this parameter is expected to better capture the dynamics of the surface wind. Globally, our analysis demonstrates regional and seasonal variations of this parameter, associated with those of wind/wave patterns. In particular, its directionality reveals the seasonal migration of intertropical convergence zone. This parameter is valuable to various further applications, for example, to help develop wind retrieval scheme from SAR measurements or to map space-time variability of ocean waves at various wavelengths.
\end{abstract}

Plain Language Summary Our paper entitled “A New Ocean SAR Cross-Spectral Parameter: Definition and Directional Property Using the Global Sentinel-1 Wave Mode Measurements" defines a new radar parameter to highlight the properties of intermediate scale ocean waves (wavelength of $15 \mathrm{~m}$ ) based on high-resolution space-borne synthetic aperture radar images. This new parameter is found to be a signed quantity, sensitive to both wind speed and direction. At global scale, we use a 1-year data to demonstrate the distribution and variation of this parameter. It is found that this parameter not only provides directional information of global winds but also exhibits distinct seasonal signatures of regional wind/wave. A case study over a polar low event also proves the capability of our new parameter in carrying surface wind information.

\section{Introduction}

Synthetic aperture radar (SAR) instruments are advanced imaging systems. In theory, the azimuth resolution can be solely specified as one half the antenna length. This results from an unambiguous tracking of the individual phase and amplitude of the return signals during the acquisition time duration. Among active microwave sensors, SARs are thus the only sensors capable of providing all-weather ocean surface observations at very high resolution to serve multipurpose ocean studies (Alpers \& Hennings, 1984; Apel \& Gonzalez, 1983; Chapron et al., 2005; Johannessen et al., 1991; Kudryavtsev et al., 2014; Jackson \& Apel, 2004). As known, the fine-Doppler analysis becomes distorted when observations are made over random time-varying sea surface. The SAR processing becomes ambiguous, and pixels are misregistered in physical space. Constructively, it helps to possibly map orbital velocity of the underlying ocean waves (Alpers \& Rufenach, 1979; Ardhuin et al., 2015). But more often, unfortunately, random orbital motions shall lead to measurable spectral cutoffs in the azimuth direction (Beal et al., 1983; Kerbaol et al., 1998; Lyzenga, 1986). The cutoff is proportional to the range-to-velocity ratio, $O(100 \mathrm{~s})$, which is systemically significant for SAR sensors aboard high altitude platforms (Lyzenga, 1986). Therefore, the possible sea state cases in which space-borne SAR image ocean wave spectra can be directly inverted into quantitative information are
(C)2019. American Geophysical Union. All Rights Reserved. 
appreciably limited, to mostly concentrate on long ocean swells (wavelength longer than $200 \mathrm{~m}$; (Ardhuin et al., 2009; Chapron et al., 2001; Collard et al., 2009) or wave propagation within the marginal ice zone (Ardhuin et al., 2017; Stopa et al., 2018) for which short-scale random orbital motions (waves with meters wavelength) have been strongly attenuated.

Still, the relatively long aperture time, $O(1 \mathrm{~s})$, used to perform the SAR processing can be uniquely exploited. Indeed, the SAR provides means to extract different ocean surface (sublooks), corresponding to different epochs, during the SAR acquisition time interval. As such, a temporal correlation analysis in the along-track can be performed between the sublooks. Resulting time shifts must then be associated to organized and coherent (during the acquisition time interval) line-of-sight motions. Such a property is currently applied to efficiently eliminate incoherent speckle noise and to help resolve the propagation ambiguity of SAR-detected swell systems, longer than the azimuth cutoff (Chapron et al., 2001; Engen \& Johnsen, 1995).

In this paper, we extend the cross-spectral analysis to investigate the time coherence of radar modulations at much smaller scales compared to swell wavelengths. The SAR cross-spectra estimate has been proposed by Engen and Johnsen (1995) to take advantage of the individual sublooks that are collected at slightly different time. In particular, the directional wave ambiguity can be resolved as well as lowering the speckle noise. We then define a new parameter called the MeAn Cross-Spectra (MACS) and is defined by filtering SAR cross-spectra within the range-detected wavelengths of 15-20 m. Given the strong dependence of these intermediate ocean waves to local wind field (Juszko et al., 1995), MACS estimates will give insight about radar modulation associated to surface waves within their equilibrium range. By definition, MACS is a complex number composed of both real and imaginary components. The imaginary part of MACS (IMACS) is expected to capture the directional signatures of intermediate waves typically corresponding to local wind fields. The normalized quantity, IMACS, is independent of any calibration issues compared to the normalized radar cross section (NRCS; Li et al., 2018). Analogous to Doppler anomaly parameter (Chapron et al., 2005; Mouche et al., 2012), it may thus be used to reduce the wind direction ambiguity in the inversion of high-resolution wind fields. In this paper, we focus on documenting the variation of IMACS relative to wind speed and direction. The global patterns of wind/waves through IMACS are also demonstrated using Sentinel-1 (S-1) WaVe mode (WV) acquisitions.

The manuscript is organized as follows. In section 2, we describe the data set used in this study as well as definition of the MACS parameter. In section 3, we present the relationship of MACS with wind field (wind speed and wind direction). Applications of MACS in local and global scale are then demonstrated and discussed in section 4. Conclusion and perspectives follow in section 5.

\section{Data Set and Definitions}

In this section, we describe S-1 WV and ancillary data used in the study. First we give the definition of the SAR cross-spectrum and then define the MACS parameter.

\subsection{S-1 Wave Mode}

The S-1 mission is a constellation of two C-band SAR sensors (S-1A and S-1B), launched by European Space Agency in April of 2014 and 2016, respectively. S-1 is the latest SAR mission in Europe after ENVISAT/ASAR (ENVIronmental SATellite / Advanced SAR). Over the open ocean, S-1 mostly operates in WV (Torres et al., 2012). Data are publicly available (https://scihub.copernicus.eu/). Each imagette is acquired every $100 \mathrm{~km}$, alternating between two incidence angles (WV1: $23.8^{\circ}$ and WV2: $36.8^{\circ}$, respectively). Compared to Envisat/ASAR capability, the size of each imagette has been increased to $20 \times 20 \mathrm{~km}$ with a 5-m spatial resolution. WV can only be operated in single polarization (VV or $\mathrm{HH}$ ). In this paper, we use data acquired by S-1A from December 2016 to November 2017 at VV polarization. To document the polarization sensitivity, HH products acquired by S-1B from 18 March to 31 June in 2017 are considered. Number of wave mode products used are listed in Table 1 . It is worth mentioning that products acquired at latitude higher than $55^{\circ}$ are filtered out to exclude possible sea-ice contamination.

We systematically co-locate (in time and space) surface winds from ECMWF (European Centre for Medium-Range Weather Forecasts) and the S-1 WV acquisitions. The ECMWF forecast winds have spatial and temporal resolution of $0.25^{\circ}$ and 3 hours, respectively. A sub-dataset consisting of upwind/downwind (wind blowing toward/along the radar look direction) cases is also considered. Specifically, the winds blowing toward $\left(0^{\circ}\right)$ or along $\left(180^{\circ}\right)$ the antenna look direction with a variation of $\pm 10^{\circ}$ are selected. The wind 
Table 1

Number of Wave Mode Products Used

\begin{tabular}{lcc}
\hline Pol & WV1 & WV2 \\
\hline VV (S-1A 2016.12-2017.11) & 350,858 & 350,842 \\
VV (S-1A 2017.03-2017.06) & 107,651 & 108,648 \\
HH (S-1B 2017.03-2017.06) & 93,915 & 94,671 \\
\hline
\end{tabular}

Note. $\mathrm{WV}=$ wave mode.

speed is then referred to as the radial wind speed, ranging from -20 to $20 \mathrm{~ms}^{-1}$. In our conventions, positive radial wind values denote upwind conditions (wind blowing toward the antenna look direction, see Figure 1). The probability density function (PDF) of co-located dataset relative to the radial wind speed is shown, top panel of Figure 1. The bin size of radial wind speed used here is $1 \mathrm{~ms}^{-1}$. The relatively nonuniform distribution of data points for intermediate wind speed ( around $7 \mathrm{~ms}^{-1}$ ) and high wind $\left(>15 \mathrm{~ms}^{-1}\right.$ ) is evident. As such, we adopt the processing method described in (Stopa \& Mouche, 2017) to equalize this sub-dataset. If there are fewer than 100 data points within a radial wind speed bin, all data points are kept. Otherwise, 100 data points are randomly selected from the dataset within that radial wind speed bin. The equalized dataset is then referred to as Data I. PDFs of Data I are plotted in bottom panel of Figure 1.

\subsection{Definitions}

\subsubsection{Estimate of SAR Image Cross-Spectra}

The method of computing SAR image cross-spectra from the single look complex (SLC) data has been thoroughly described in Johnsen and Collard (2009). A simplified flowchart describing the processing is given in Figure 2a. The S-1 lookup tables are applied to calibrate the SLC products. Two subsequent preprocessing steps are also performed to eliminate the signal of bright targets. The procedure "detrending" removes the low-frequency (nonwave) signatures by low-pass filtering the input image (Johnsen \& Collard, 2009). Then, each SLC imagette is split into tiles of $512 \times 512$ pixels. From each tile, a fast Fourier transform (FFT) is performed, and the resulting azimuthal (slow-time) spectrum is divided into three nonoverlapping parts as in Figure 2b. The sublook intensity images are obtained by applying an inverse FFT to each one of these parts. Finally, cospectra and cross-spectra are defined as

$$
P_{s}^{(m, n)}(\mathbf{k}, \Delta t)=\frac{\left\langle\mathcal{F}\left[I^{(m)}(\mathbf{x})\right] \cdot \mathcal{F}^{*}\left[I^{(n)}(\mathbf{x})\right]\right\rangle}{\left\langle I^{(m)}(\mathbf{x})\right\rangle \cdot\left\langle I^{(n)}(\mathbf{x})\right\rangle}-\delta(\mathbf{k}), m, n \in[1,2,3],
$$

where $\Delta t=|m-n| \tau$ is separation time between $m$ th and $n$th sublooks and $\tau$ is the time interval between two adjacent looks. In this paper, $\tau=0.27 \times T_{a}$ with $T_{a}$ being SAR integration time. $\left\langle I^{(m)}(\mathbf{x})\right\rangle$ denotes the mean intensity of the $m$ th look, $\mathcal{F}[\cdot]$ stands for the two-dimensional FFT operator, and the superscript $*$ is complex conjugate. $\delta(\mathbf{k})$ is a Dirac Delta function. The cospectrum is obtained when $m=n$. Computed coand cross-spectra for all tiles are then averaged to reduce speckle noise, leading to one cospectra and possibly several cross-spectra (obtained for different values of $\Delta t$ ) for each WV image. This averaging technique

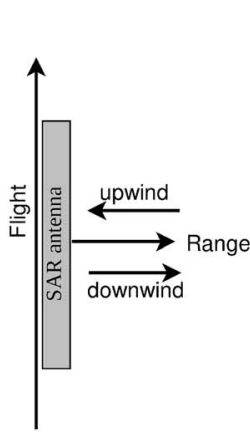

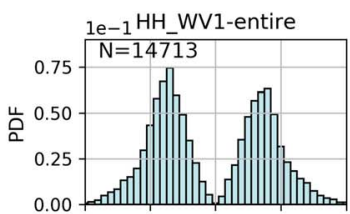

1e-2 $\mathrm{HH}_{-}$WV1 Data I

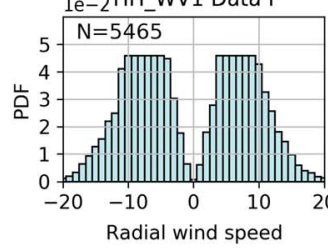

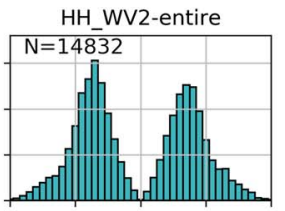

HH_WV2 Data I

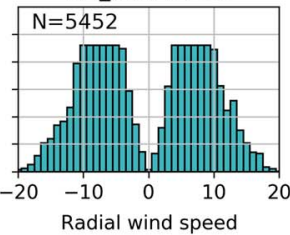

W_WV1-entire

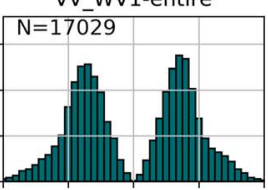

V_WV1 Data I

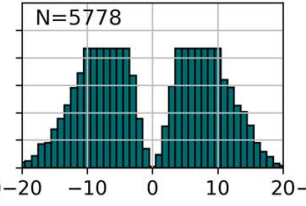

VV_WV2-entire

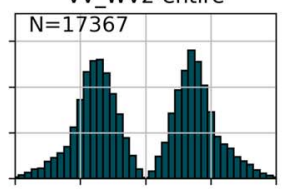

VV_WV2 Data I

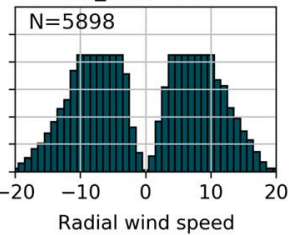

Figure 1. (left) Probability density functions (PDFs) of the synthetic aperture radar (SAR) wind speeds. (top) PDF of collocated radial wind speed between Sentinel-1A/B and European Centre for Medium-Range Weather Forecasts for HH and VV, WV1 and WV2, respectively. (bottom) PDF of correspondingly equalized data set (denoted as Data I). The wind speed bin used here is $1 \mathrm{~m} / \mathrm{s}$. Data with wind greater than $20 \mathrm{~m} / \mathrm{s}$ has been excluded due to lack of enough points. Positive radial winds correspond to upwind direction. 

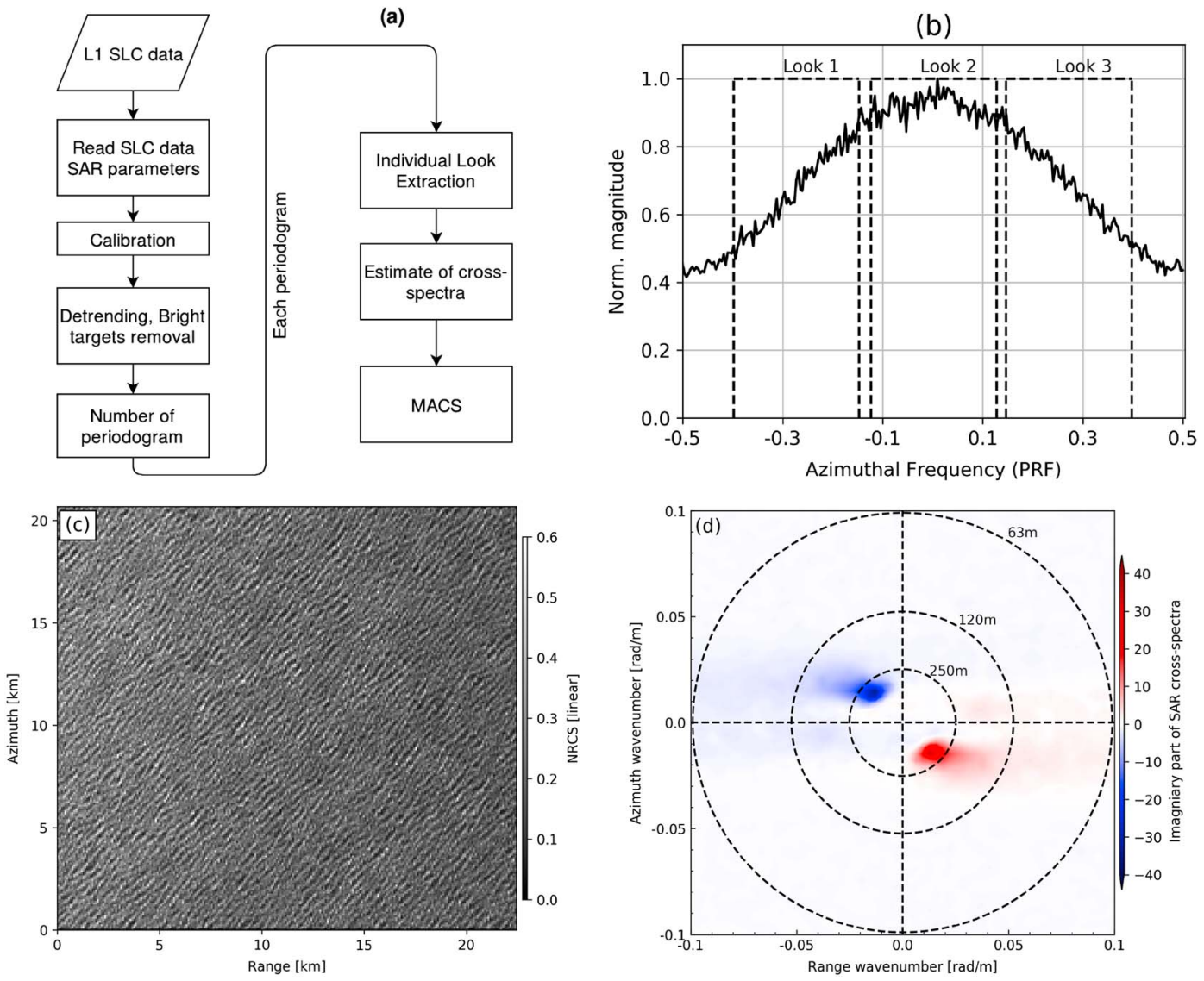

Figure 2. (a) Flowchart of synthetic aperture radar (SAR) image cross-spectral processing; (b) simplified view of individual look extraction based on Doppler bandwidth spectrum. (c) An example of SAR image acquired by Sentinel-1A. Incidence angle is $23.8^{\circ}$. (d) The corresponding imaginary part of SAR cross-spectra computed for $\Delta t=2 \tau$. See text for details. SLC $=$ single look complex; PRF $=$ Pulse Repetition Frequency .

is referred to as periodogram in the flowchart. Figure 2c shows a SAR image acquired by S-1A WV1 and the corresponding imaginary part of SAR cross-spectra for $\Delta t=2 \tau$ in Figure $2 \mathrm{~d}$. The imaginary part has opposite signs at the symmetric peaks, which is key to resolving the ocean waves propagation ambiguity.

2.2.2. MACS of Intermediate Waves

Formally, the SAR nonlinear mapping is

$$
I_{S A R}(\mathbf{x})=\int I_{R A R}\left(\mathbf{x}^{\prime}\right) \delta\left(\mathbf{x}-\mathbf{x}^{\prime}-\xi\left(\mathbf{x}^{\prime}\right)\right) d \mathbf{x}^{\prime}
$$

with $I_{R A R}$ the initial detected real aperture radar (RAR) intensity. $\xi$ random shifts, and $I_{S A R}$ the resulting SAR intensity. While this mapping is nonlinear, it still ensures the conservation of the overall mean image intensity, that is, $\int I_{S A R}() d \mathbf{x}=\int I_{R A R}(\mathbf{x}) d \mathbf{x}$. Accordingly, using a spatial smoothing along the azimuth direction should suppress undesirable nonlinear SAR processing effects. Conveniently, this filtering is carried out in the spectral domain by reducing the azimuthal wave number domain $(<2 \pi / 600 \mathrm{rad} / \mathrm{m})$. The MACS parameter is then defined over intermediate range-detected waves, corresponding to filtering the cross-spectra around range (across-track) wavelength between 15 and $20 \mathrm{~m}$. The lower limit of range wavelength is set to be approximately three times the nominal S-1 line-of-sight ground resolution. It ensures that modulations of interest are sufficiently well resolved. A schematic view for this spectral filtering to extract intermediate range waves, and to best minimize the SAR azimuthal distortion, is shown in Figure 3. The MACS over area $\mathbf{A}$ is

$$
\operatorname{MACS}(\bar{k}, \Delta t)=\frac{1}{N} \sum_{n=1}^{N} P_{s}^{(m, n)}\left(k_{a z}, k_{r a}, \Delta t\right),\left(k_{a z}, k_{r a}\right) \in \mathbf{A}
$$




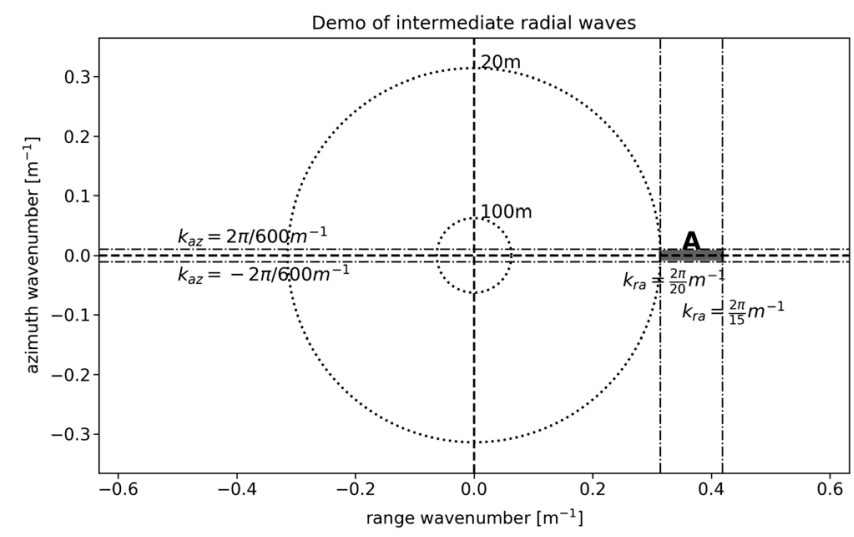

Figure 3. A schematic view of intermediate radial waves denoted by $\mathbf{A}$ over the mean cross-spectra is estimated. The limits of wavelength used in this study is $600 \mathrm{~m}$ in azimuth and between 15 and $20 \mathrm{~m}$ in range. The dotted circles represent wavelengths of 100 and $20 \mathrm{~m}$ from inner to outer.

where $N$ is number of points within area $\mathbf{A}$. The cross-spectra can be calculated for different combinations of sublooks. Throughout rest of this paper, we only use MACS defined based on the SAR cross-spectra computed between two adjacent sublooks, denoted as MACS ${ }^{(1)}$. In terms of the cross-spectral transformation (Engen \& Johnsen, 1995), MACS ${ }^{(1)}$ carries both magnitude and phase information of radar backscatter modulations. Neglecting the highly reduced contribution from velocity bunching, MACS describes the intermediate ocean wave spectra weighted by the RAR modulation transfer function (MTF). MACS therefore contains information related to different imaging contributions (hydrodynamic and tilt) for given incidence angle and polarization. In this study, we solely concentrate on the imaginary component of MACS, denoted as $\operatorname{IMACS}^{(1)}$.

\section{Characteristics of IMACS ${ }^{(1)}$}

\subsection{Directional IMACS ${ }^{(1)}$}

IMACS $^{(1)}$ is displayed relative to radial wind speed in Figure 4. Under developed sea states, propagation of intermediate waves should correspond with the local wind direction (Juszko et al., 1995; Quilfen et al., 1999). As anticipated, IMACS ${ }^{(1)}$ changes sign when the wind direction reverses. Statistically, it is positive for the downwind direction and negative for the upwind.

To first order, it is a linear function of radial wind speed, up to $\pm 10 \mathrm{~m} / \mathrm{s}$. For WV1 in Figures $4 \mathrm{a}$ and $4 \mathrm{c}, \operatorname{IMACS}^{(1)}$ has nearly the same slope for upwind and downwind directions. For WV2 in Figures $4 \mathrm{~b}$ and $4 \mathrm{~d}$, $\operatorname{IMACS}^{(1)}$ is more sensitive under upwind conditions than for downwind conditions. For a given radial wind, $\operatorname{IMACS}^{(1)}$ decreases with the increasing incidence angle. At given incidence angle, IMACS ${ }^{(1)}$ obtained in $\mathrm{HH}$ polarization is larger than in VV. This is expected, as the HH MTFs have generally been reported larger than VV MTFs, expressing a larger sensitivity to the hydrodynamic and tilt modulation effects (Alpers et al., 1981; Kudryavtsev et al., 2014). A slight saturation of $\operatorname{IMACS}^{(1)}$, around wind speed higher than $10 \mathrm{~m} / \mathrm{s}$, is apparent for WV1, but not for WV2.

Quite remarkably, as shown in Figure 4a, IMACS parameters share similar characteristics with the Doppler centroid anomaly (DCA; Chapron et al., 2005; see their Figure 5). Indeed, both parameters are directly linked to the temporal evolution and coherency of detected sea surface scatters. The DCA encompasses all detected scales, and estimates are performed using cross-correlation between consecutive (in time) complex signals. For IMACS, it is the coherency and time evolution of filtered reflectively modulations that are captured. As such, the cross-correlation can apply between the intensity signals acquired at larger time differences. For IMACS, the cross-correlation will measure the coherency and time evolution of the filtered modulations of the intensity signals. Under a two-scale scattering model decomposition (see Appendix B in Chapron et al., 2005; also equation (3) in Johannessen et al., 2008), such intermediate scale time evolution contribute to the DCA parameter. To first order, both parameters, DCA and IMACS, must be strongly linked, closely tracing the wind direction within a single SAR image. To date, the lack of accurate satellite attitude and orbital control system information hampers the S-1 DCA interpretation, as the geometric DC (Doppler Centroid ) accuracy is not sufficient. This is not as stringent for $\operatorname{IMACS}^{(1)}$. A precise time or Doppler origin is not required, 

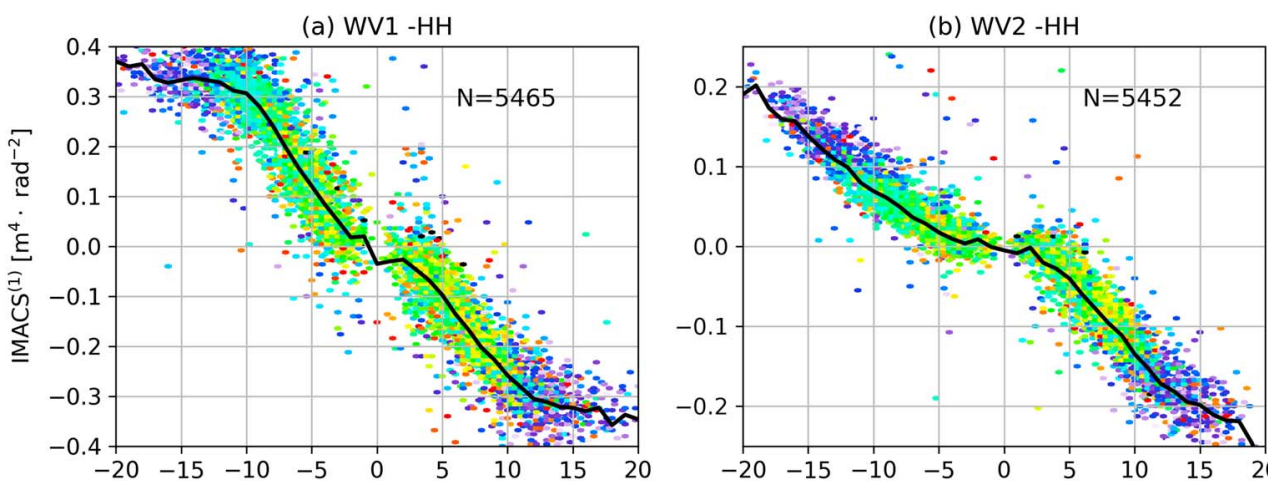

$-40$

(c) WV1 -VV

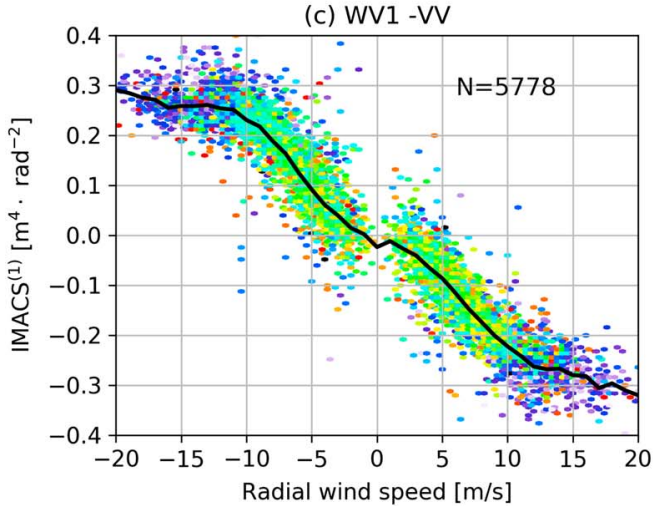

(d) WV2 -VV

Figure 4. IMACS ${ }^{(1)}$ relative to the radial wind speed for (a) WV1, HH; (b) WV2, HH ; (c) WV1, VV; (d) WV2, VV based on the equalized Data I. The color represents latitude. The solid black line marks mean curve to the observations. Positive radial wind speeds denote upwind configuration. IMACS = imaginary part of mean cross-spectra.

as the $\operatorname{IMACS}^{(1)}$ can isolate the ocean scene time evolution by matching detected (and filtered) modulations of intensity signals. Accordingly, IMACS $^{(1)}$ can therefore be an alternative to DCA measurements to help constrain the ocean surface wind vector inversion at moderate to high spatial resolution (Mouche et al., 2012).

\subsection{Wind Direction Dependency of $\operatorname{IMACS}^{(1)}$}

In this section, the $\operatorname{IMACS}^{(1)}$ dependency on wind direction is demonstrated. For simplicity, only acquisition of WV1 at VV polarization are analyzed. Figure 5a displays the $\operatorname{IMACS}^{(1)}$ relative to wind direction at wind speed of $7 \mathrm{~m} / \mathrm{s}$ (dots). The mean is marked as solid curve. It closely follows a cosine function across the entire wind direction range. $\operatorname{IMACS}^{(1)}$ reaches the minimum at upwind $\left(0^{\circ}\right)$, the maximum at downwind $\left(180^{\circ}\right)$. Again, azimuthal modulation of IMACS ${ }^{(1)}$ is found similar to that of DCA, as shown in Mouche et al. (2012). Analogous to the DCA (see Figure 2e in Mouche et al., 2012), a wind retrieval algorithm combining both the NRCS and IMACS ${ }^{(1)}$ would greatly benefit from the new radar parameter, adding a constraint (directly derived from SAR measurement) to the inverse problem.

As defined, IMACS ${ }^{(1)}$ is defined to extract information close to the radar range direction, precisely filtering over $\pm 2 \pi / 600 \mathrm{rad} / \mathrm{m}$ along the azimuth direction in Figure 3. To help discriminate the contributions between azimuthal quadrants, IMACS ${ }^{(1)}$ is then further decomposed into two parts. The part defined over $[-2 \pi / 600 \mathrm{rad} / \mathrm{m}, 0]$ is denoted as $\operatorname{IMACS}_{-A}^{(1)}$, and the other over $[0,2 \pi / 600 \mathrm{rad} / \mathrm{m}]$ is $\operatorname{IMACS}_{+A}^{(1)}$. For wind speed at $7 \mathrm{~m} / \mathrm{s}$, these two parameters are reported in Figure 5a. Estimates are different, especially for the two crosswind directions $\left(90^{\circ}\right.$ and $\left.270^{\circ}\right)$. At $90^{\circ}, \operatorname{IMACS}_{+A}^{(1)}$ (dashed line) is almost identical to IMACS ${ }_{-A}^{(1)}$ (dot-

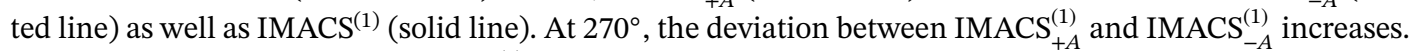
Variation of their deviation $\triangle \mathrm{IMACS}_{ \pm}^{(1)}$ versus wind direction is accordingly quantified in Figure $5 \mathrm{~b}$. Notice that $\Delta \operatorname{IMACS}_{ \pm}^{(1)}$ exhibits distinct azimuthal modulation than $\operatorname{IMACS}^{(1)}$. It is close to zero at crosswind of $90^{\circ}$ and decreases to its minimum at $270^{\circ}$. This contrast between two crosswinds increases with wind speed as shown in Figure $5 \mathrm{c}$. At low wind speed $(<3 \mathrm{~m} / \mathrm{s})$, ocean wave features are not sufficiently well resolved due to low signal-to-noise ratio. This can explain the low contrast of $\Delta \operatorname{IMACS}_{ \pm}^{(1)}$ across wind directions at 

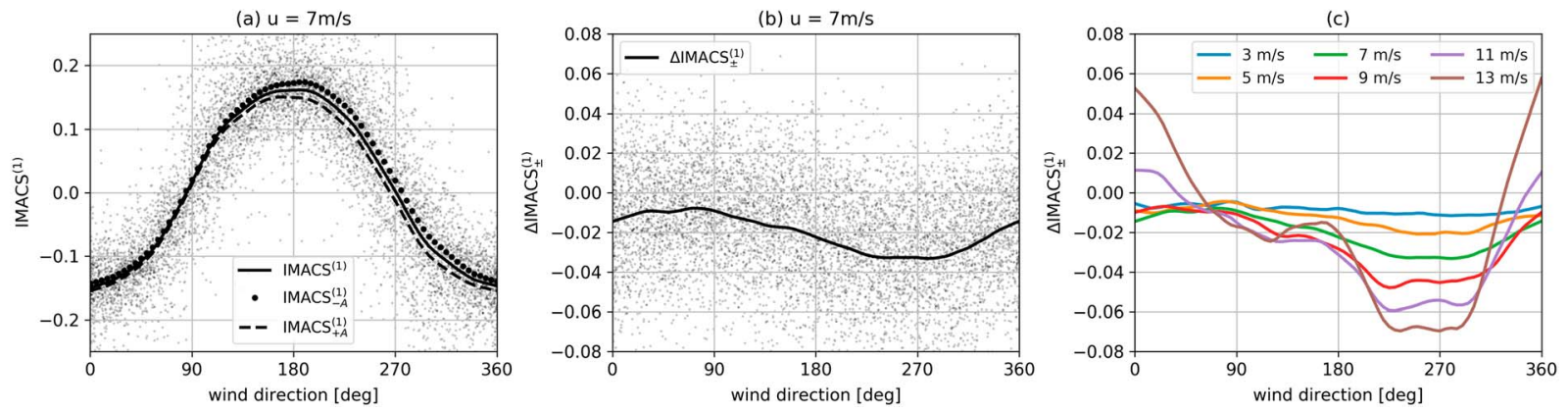

Figure 5. (a) Scatter plot of $\operatorname{IMACS}^{(1)}$ relative to wind direction at wind speed of $7 \mathrm{~m} / \mathrm{s}^{\text {s. Binned average of IMACS }}{ }^{(1)}$ (solid), IMACS + I $_{+A}$ (dashed), and IMACS ${ }_{-A}^{(1)}$ (dotted) is also plotted. (b) $\Delta \operatorname{IMACS}_{ \pm}^{(1)}$ IMACS $_{+A}^{(1)}-\operatorname{IMACS}_{-A}^{(1)}$ ) at $7 \mathrm{~m} / \mathrm{s}$. (c) $\Delta \operatorname{IMACS}_{ \pm}^{(1)}$ relative to wind direction for various wind speeds. Wind direction of $0^{\circ}$ denotes upwind. IMACS $=$ imaginary part of mean cross-spectra.

$3 \mathrm{~m} / \mathrm{s}$. With increasing wind speed, the contrast becomes pronounced. This distinguished wind direction dependence can thus help resolve the wind direction ambiguities, to provide means to obtain one single wind vector. The wind inversion scheme using $\Delta \mathrm{IMACS}_{ \pm}^{(1)}$ is beyond scope of the present paper. Present efforts are underway Nilsen et al. (2018) and left to further validation efforts.

\section{Applications}

\subsection{IMACS ${ }^{(1)}$ Over a Polar Low}

Statistically, Figure 4, IMACS ${ }^{(1)}$ parameters capture the radial wind information. Hereafter, a particular localized event is more precisely analyzed. A polar low event is chosen, as they are difficult to detect using the visual remote sensing. Microwave scatterometers, on the other hand, are advanced but limited by the coarse resolution $(12.5 \mathrm{~km})$. SAR measurements are therefore of practical use to yield high-resolution structures of the local wind field (Furevik et al., 2015).

Figure 6a presents a SAR image of C-band HH NRCS over a polar low event. Data have been acquired by S-1A, in the interferometric wide swath mode, on 29 December 2016 at 15:03 UTC. The incidence angle at near range is $41.2^{\circ}$ and $45.5^{\circ}$ at far range. The ECMWF forecast winds at $15 \mathrm{~h} 00 \mathrm{UTC}$ are superimposed to complement the NRCS. This event was an intense mesoscale extratropical cyclone, quite typical over the oceans at high latitude in cold polar areas (Montgomery \& Farrell, 1992; Smirnova et al., 2015). In the Northern Hemisphere, the winds rotate counterclockwise. Considering the SAR flight direction, toward the North in this case, it corresponds to the upwind conditions (blowing toward the antenna pointing direction) in the northern sector and, subsequently, the downwind conditions in the southern sector. Given the distinct up-downwind asymmetry of NRCS at this incidence angle, a darker pattern is indeed found in the southern sector. However, at this point, it is difficult to interpret the wind variation without use of any external ancillary information, such as the ECMWF wind direction. By comparison, $\operatorname{IMACS}^{(1)}$ parameters, mapped on Figure 6b, clearly provide the first-order directional information of local winds. The opposite sign of
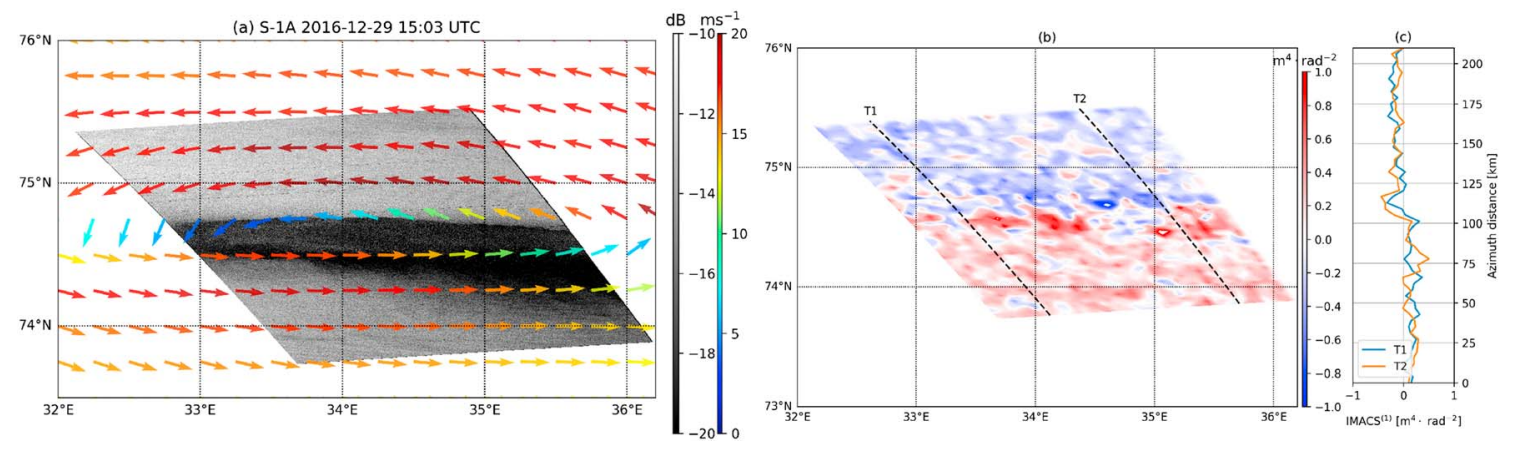

Figure 6. (a) Normalized radar cross-section representation acquired by S-1A interferometric wide swath on 29 December 2016 at 15:03 UTC over a polar low. The European Centre for Medium-Range Weather Forecasts forecast winds at 15:00 UTC are superimposed as arrows with colors denoting the wind speed. (b) IMACS ${ }^{(1)}$ computed over moving windows. (c) IMACS ${ }^{(1)}$ along two transects depicted by the two black lines in plot (b). S-1 = Sentinel-1; IMACS = imaginary part of mean cross-spectra. 


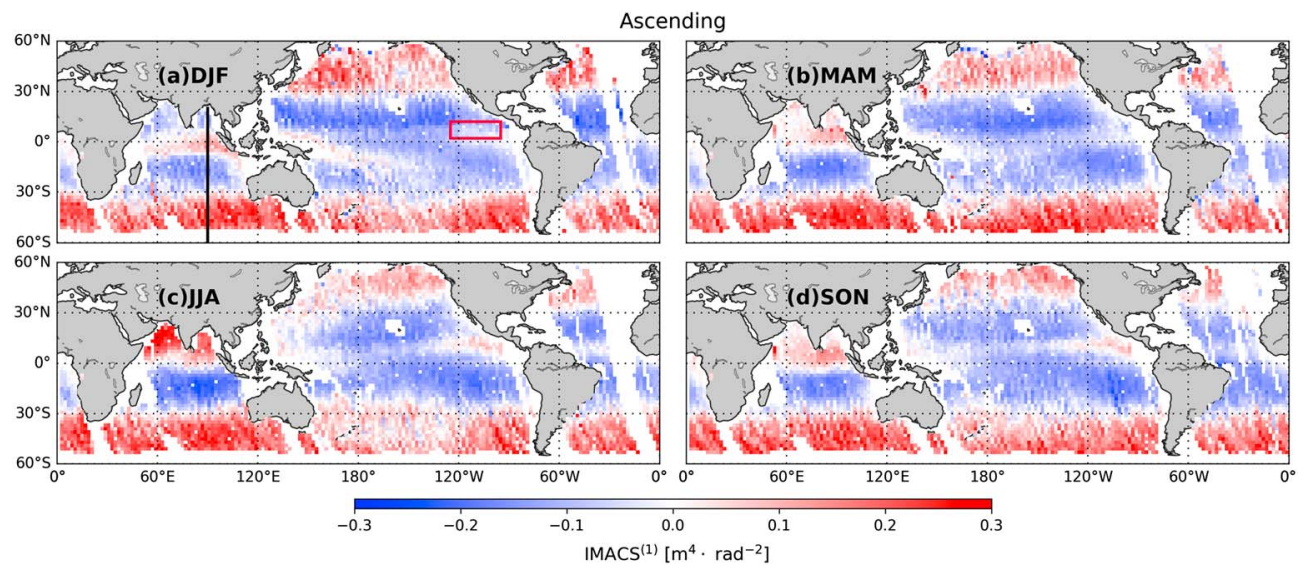

Figure 7. Seasonal global map of $\operatorname{IMACS}^{(1)}$ based on the data acquired by S-1A WV1 over ascending pass from December 2016 to November 2017. The negative IMACS $^{(1)}$ corresponds to upwind direction with wind roughly blowing from the east to the west. Data are shown at bin of $2^{\circ}$ in both longitude and latitude. The red rectangle in the East Pacific Ocean and vertical transect in the Indian Ocean are marked as areas of interest for the following analysis. S-1 = Sentinel-1; IMACS = imaginary part of mean cross-spectra; DJF = December-January-February; JJA = June-July-August; MAM = March-April-May; SON = September-October-November.

IMACS $^{(1)}$ (blue to red) well captures the reversed wind direction as in Figure 6a. This is further demonstrated by the averaged $\operatorname{IMACS}^{(1)}$ along the two transects in Figure 6c. IMACS ${ }^{(1)}$ at transect "T1" and "T2" has the same order of magnitude, corresponding to the comparable wind speed and direction as given by ECMWF winds. In addition, IMACS ${ }^{(1)}$ magnitude is roughly consistent with that in Figure $4 \mathrm{~b}$ (different incidence angles). This indicates that the IMACS ${ }^{(1)}$ derived from the global analysis can also be applied to wide swath mode. It should also be noted that over the central part (roughly along the latitude $75^{\circ} \mathrm{N}$ ), $\operatorname{IMACS}^{(1)}$ is not trustworthy, likely due to the low signal-to-noise ratio.

\subsection{Climatological Analysis of IMACS ${ }^{(1)}$}

As demonstrated, the IMACS ${ }^{(1)}$ is complementary to the conventional NRCS measurements. Free from any calibration issues, IMACS ${ }^{(1)}$ essentially contains information related to the coherent time evolution of filtered radar modulations. The IMACS ${ }^{(1)}$ captures not only the local wind features but also the intermediate scale wave features. Seasonal average of $\operatorname{IMACS}^{(1)}$ is presented in Figure 7, grouped as June-July-August (JJA), September-October-November (SON), December-January-February (DJF), and March-April-May (MAM). S-1A WV1 acquisition from December 2016 to November 2017 over ascending pass is considered. The white areas are due to insufficient number of acquisitions. The regions with missing data are close to coastlines and over the northeast Atlantic Ocean where other acquisition modes (interferometric wide swath and Extra Wide swath) are operated. The same convention as in Figure 4 is used; the negative values correspond to the upwind direction (wind blowing from the east to the west).

In the tropics $\left(30^{\circ} \mathrm{S}\right.$ to $\left.30^{\circ} \mathrm{N}\right)$, the prevailing trade winds are blowing from east to west. It thus corresponds to upwind conditions for S-1 ascending pass, resulting in consistently negative IMACS ${ }^{(1)}$ values. The migration of the intertropical convergence zone (ITCZ) is clearly well captured by the seasonal sign changes of IMACS $^{(1)}$. For JJA (Figure 7c) and SON (Figure 7d), the ITCZ is located in north of the Equator, about latitude $10^{\circ} \mathrm{N}$, extending to the West Pacific Ocean. For the other two seasons, the ITCZ moves southward and is located near the Equator with reduced zonal extension.

At the midlatitudes $\left(3-60^{\circ}\right)$, the wind predominately blow from the west to the east, that is, downwind conditions. Accordingly, the positive IMACS ${ }^{(1)}$ are obtained. The Southern Hemisphere extratropics are very consistent with high magnitude of IMACS ${ }^{(1)}$. This is associated to the regular storms over this area throughout the year. For the same reason, we observe the maximum values of IMACS ${ }^{(1)}$ in the northwest Pacific Ocean in season DJF (Figure 7a), associated with the strongest winter storms. Lack of data precludes this feature to appear over the Atlantic Ocean. It is clear that boundaries between the trade winds and westerlies vary with seasons: The boundary migrates southward from JJA to DJF, consistent with the motion of ITCZ.

To further document the ITCZ migration captured by $\operatorname{IMACS}^{(1)}$, a regional analysis is performed. It corresponds to the region delineated by a red rectangle in Figure $7 \mathrm{a}$, extending over the east Pacific Ocean $\left(2-12^{\circ} \mathrm{N}\right.$ 

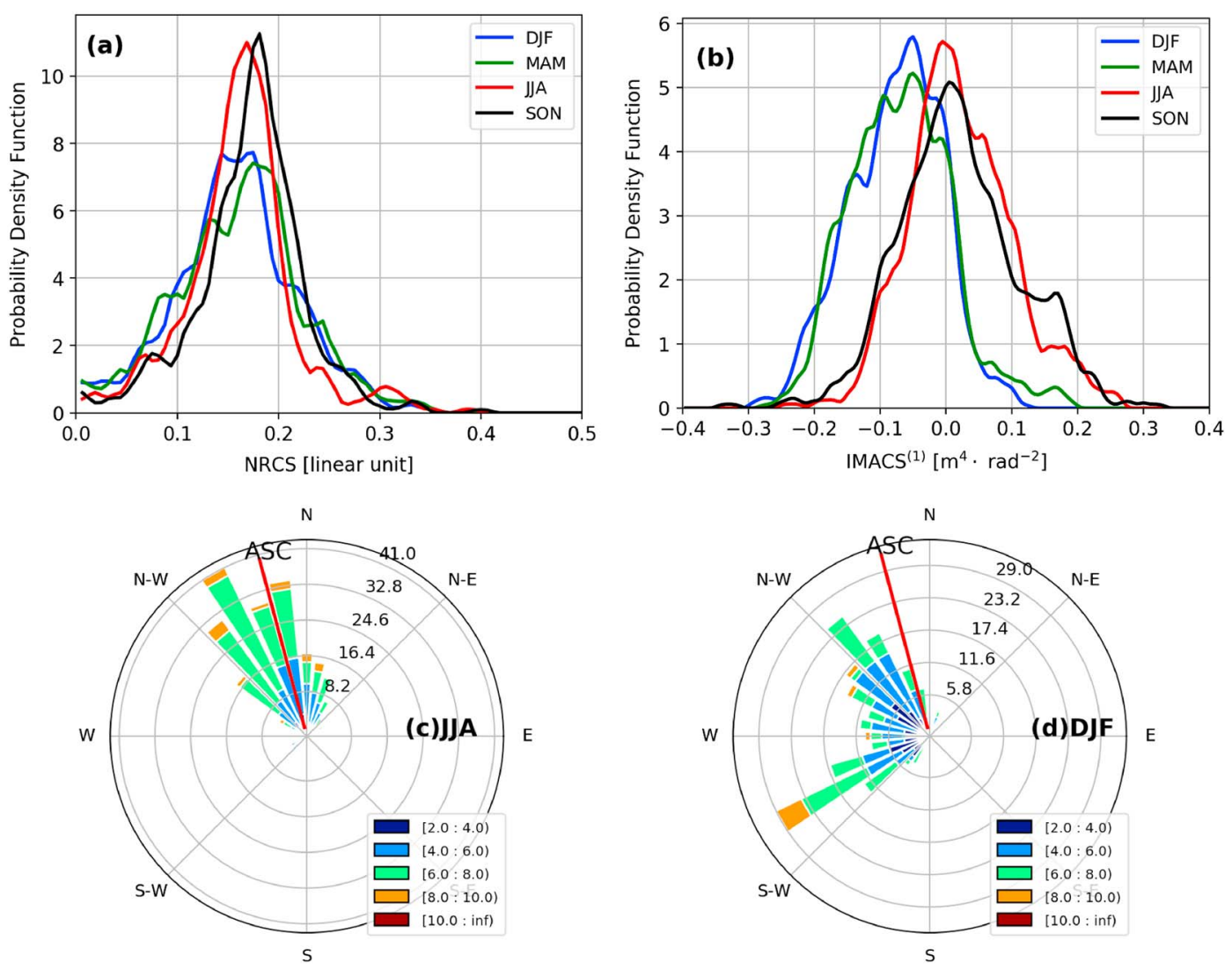

Figure 8. Probability density function of (a) the NRCS and (b) the IMACS ${ }^{(1)}$ over the east Equatorial Current area (marked by red rectangle in Figure 7a) are presented for four seasons. The wind rose of collocated European Centre for Medium-Range Weather Forecasts winds is also depicted for (c) season of JJA; (d) season DJF. NRCS = normalized radar cross section; IMACS = imaginary part of mean cross-spectra; DJF = December-January-February; JJA = June-July-August; MAM = March-April-May; SON = September-October-November; ASC = Ascending.

in latitude and $125-95^{\circ} \mathrm{W}$ in longitude). Seasonal PDF of NRCS as well as IMACS ${ }^{(1)}$ are shown in Figures $8 \mathrm{a}$ and $8 \mathrm{~b}$, respectively. From the PDF of NRCS, the maximum density corresponds with the seasons, being around 0.1687 (linear unit). The wider PDF in DJF and MAM implies the larger spread of local winds (i.e., more variability) but gives no further details. On the contrary, PDF of the IMACS ${ }^{(1)}$ greatly differs from JJA to DJF. The maximum point in JJA and SON is located around +0.010 , suggesting the prevailing winds to have small zonal components. For DJF and MAM, the maximum shifts toward -0.055 , corresponding to the winds blowing from the east. The PDFs of IMACS ${ }^{(1)}$ have larger spread for DJF/MAM than for JJA/SON. Given the analogy between JJA (DJF) and SON (MAM), wind rose of the collocated ECMWF forecast winds is displayed for JJA in Figure 8c and DJF in Figure 8d. During JJA, most of the local winds blow along the SAR ascending flight direction, resulting in nearly zero IMACS ${ }^{(1)}$, Figure 8b. For DJF, the winds blowing from the East are predominant and have large spread. Based on this analysis, the NRCS measurements are not sufficient to manifest the seasonal migration of ITCZ. But combined NRCS and IMACS ${ }^{(1)}$ may open new strategies to determine the global wind/wave climatologies.

Over the Indian Ocean, there is also a distinct seasonality captured by the $\operatorname{IMACS}^{(1)}$. North of the equator, IMACS $^{(1)}$ is negative in DJF/MAM, positive in JJA/SON. The sign change is to be attributed to the reversed wind direction during the monsoon season (usually occurring from July to October). A different rate of sign changes of IMACS ${ }^{(1)}$ is well captured from north to south, across the Indian Ocean. A transect at the longitude $90^{\circ} \mathrm{E}$ is chosen to demonstrate this seasonal pattern of IMACS ${ }^{(1)}$. The seasonal average of IMACS ${ }^{(1)}$ and wind direction along the transect are given in Figures 9a and 9b, respectively. During JJA (red curve) and SON (black curve), IMACS ${ }^{(1)}$ decreases from positive to negative at latitude $5^{\circ} \mathrm{S}$ to reaches the negative maximum at $18^{\circ} \mathrm{S}$. It then starts to increase from negative to the positive maximum at $50^{\circ} \mathrm{S}$. Marked features of IMACS ${ }^{(1)}$ during DJF (red line) and MAM (green line) are in north of the equator. During DJF, IMACS ${ }^{(1)}$ is 

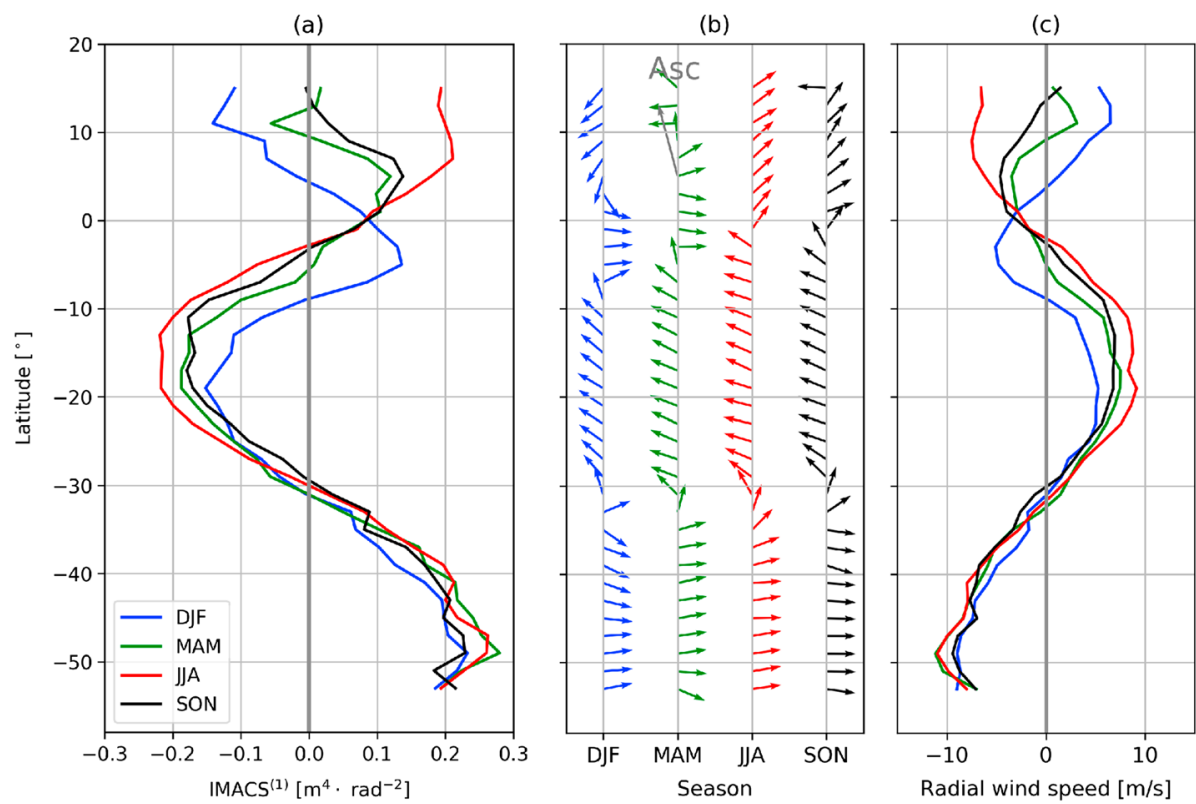

Figure 9. Seasonal average of (a) $\operatorname{IMACS}^{(1)}$, (b) collocated European Centre for Medium-Range Weather Forecasts wind direction, and (c) the projected radial wind speed relative to latitude along the transect of $90^{\circ} \mathrm{E}$ shown as vertical black line in Figure 7a. Data are averaged over latitude bin of $2^{\circ}$. The gray arrow denotes synthetic aperture radar flight direction over ascending pass. Wind convention is the same with Figure 7. IMACS = imaginary part of mean cross-spectra; DJF = December-January-February; JJA = June-July-August; MAM = March-April-May; $\mathrm{SON}=$ September-October-November.

first negative and then inverses its sign, becoming positive at latitude $3^{\circ} \mathrm{N}$. The sign change from positive to negative more precisely occurs at latitude $9^{\circ} \mathrm{S}$. The $\operatorname{IMACS}^{(1)}$ variation is consistent with the wind direction change shown by the projected radial ECMWF winds in Figure 9c.

This strong seasonal variation of IMACS ${ }^{(1)}$ is further quantified by the interseasonal variability, Figure 10. It is computed as the standard deviation of seasonal average of IMACS ${ }^{(1)}$. A larger variability of $\operatorname{IMACS}^{(1)}$ occurs over the Northern Hemisphere. Around $40^{\circ} \mathrm{N}$ in Northern Hemisphere, the largest seasonal variability is estimated. In the Southern Hemisphere, $\operatorname{IMACS}^{(1)}$ parameters have relatively smaller standard deviation at $50^{\circ} \mathrm{S}$. By contrast, the $\operatorname{IMACS}^{(1)}$ is largely variable, throughout the year, at longitude between $160^{\circ} \mathrm{W}$ and $130^{\circ} \mathrm{W}$. The Indian monsoon region also presents a higher IMACS ${ }^{(1)}$ variance. This is caused by the reversed wind directions during the monsoon season, Figure 7. By comparison, the trade wind regions over the Pacific Ocean display much lower variability. A band of increased variability is evident, centered

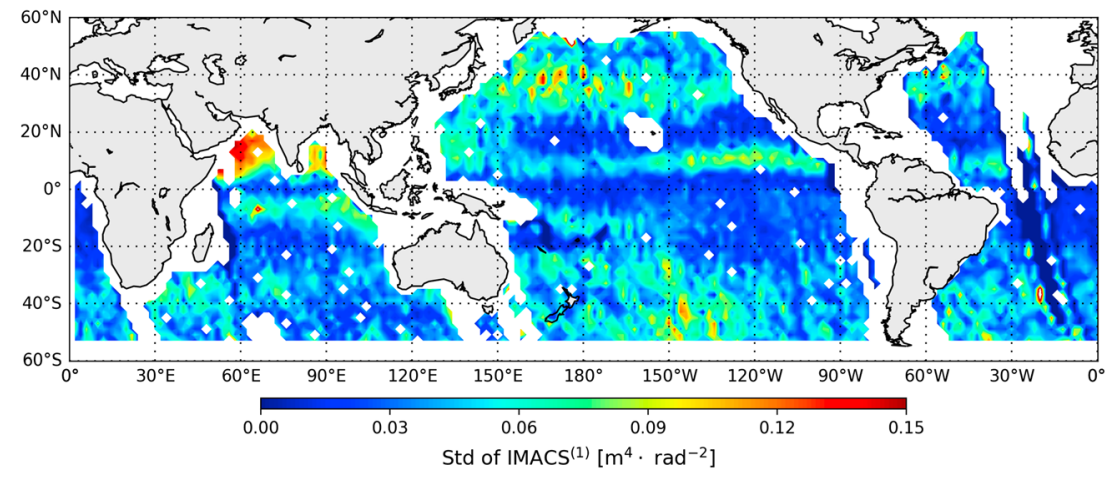

Figure 10. The interseasonal standard deviation of $\operatorname{IMACS}^{(1)}$ computed from seasonal average given in Figure 7. IMACS = imaginary part of mean cross-spectra. 
at $10^{\circ} \mathrm{N}$ on the Pacific Ocean. It corresponds to the wind field changes over the ITCZ, Figure 8 . This band extends to the west Pacific Ocean. Over the Indian Ocean, a zonal band of distinct standard deviation, centered at $10^{\circ} \mathrm{S}$, is also associated to the wind direction changes, Figure 9.

\section{Conclusion and Perspectives}

In this paper, we isolate the nondistorted wavelengths of 15-20 m associated with wind and wave motions. The larger spatial footprint and higher spatial resolution of S-1 wave mode allows us to analyze these intermediate components relative to its predecessor ENVISAT/ASAR. We then propose a new parameter called MACS, which responds to both the wind speed and direction.

For the upwind or downwind conditions, a signed and nearly linear variation is obtained. This result bears strong similarity with the DCA sensitivity to radial wind speed (see Figure 5b in Chapron et al., 2005). We expect that the MACS captures the overall correlation between the surface wave motions and the filtered (radar) detected contrasts, in line with a two-scale interpretation of the wave motion bias associated to DCA measurements (see equation (B1) in Chapron et al., 2005, and equation (3) in Johannessen et al., 2008). Comparable to the previous developments (Mouche et al., 2012), such dependencies are complementary to the NRCS measurements and open for new analysis strategies. As first anticipated and demonstrated with a polar low case, these two high-resolution radar quantities, that is, NRCS and MACS (possibly DCA when available), should be advantageously used to help constrain the local wind retrieval schemes from SAR measurements. Providing improved local wind estimates will also have positive impact on SAR-wave retrieval algorithms to more precisely inform the local sea state conditions.

Moreover, given the close dependency of MACS to the strength of the local radial wind component, MACS may also have the potential to directly help remove the wave bias contribution from DCA estimates. This step is essential to recover the geophysical Doppler information solely related to ocean surface current. In that context, MACS estimates can help bypass the need of a priori wind information (e.g., Rouault et al., 2010). Such a demonstration is beyond the scope of the present paper and will be the focus of future studies, possibly revisiting the Envisat/ASAR mission. This can also help to establish basis of algorithms in order to retrieve oceanic current and wind speed from future ocean concept missions. From its definition, MACS parameter can also be refined to encompass bifrequency or bilocation beam instrumental configuration, as well as highly range resolved RAR measurements (Ardhuin et al., 2018; Nouguier et al., 2018).

Finally, as further demonstrated in the present study, global mapping and analysis of MACS can efficiently quantify regional and seasonal wind and (intermediate) wave properties and space-time variability. As such, this new parameter can be used to study the wind/wave climate from a new perspective. For example, this aspect will lead to revisit the available 10-year archive of ENVISAT/ASAR wave mode data. Given a relatively coarser resolution $(10 \mathrm{~m}$ ) compared to $\mathrm{S}-1$, the filtering operation shall certainly be adapted, that is, possibly down to 50-m scales. This new parameter can also be used to jointly analyze wind and equilibrium wave range climate by considering an adaptive definition over various wavelengths. Still, for moderate to high wind conditions, MACS parameters will likely carry valuable information related to radial wind conditions to enhance previous attempts (Stopa et al., 2015) to more precisely document long-term wind/wave climate properties.

Acknowledgments

This work was supported by European Space Agency through S1-4SCI Ocean Study project (contract

4000115170/15/I-SBo), Sentinel-1 A

Mission Performance Center (contract 4000107360/12/I-LG), SATROSS

project (contract 4000120680), and by CNES (TOSCA program, COWS

project). Copernicus Sentinel data (2016 and 2017) are used. The ECMWF forecast winds are obtained in the framework of Sentinel-1 A Mission Performance Center ESA project and are publicly available (ecmwf.int). Huimin Li also acknowledges $\mathrm{PhD}$ financial support from the China Scholarship Council (CSC).

\section{References}

Alpers, W., \& Hennings, I. (1984). A theory of the imaging mechanism of underwater bottom topography by real and synthetic aperture radar. Journal of Geophysical Research, 89(C6), 10,529-10,546. https://doi.org/10.1029/JC089ic06p10529

Alpers, W. R., Ross, D. B., \& Rufenach, C. L. (1981). On the detectability of ocean surface waves by real and synthetic aperture radar. Journal of Geophysical Research, 86(C7), 6481-6498. https://doi.org/10.1029/JC086iC07p06481

Alpers, W., \& Rufenach, C. (1979). The effect of orbital motions on synthetic aperture radar imagery of ocean waves. IEEE Transactions on Antennas and Propagation, 27(5), 685-690.

Apel, J. R., \& Gonzalez, F. I. (1983). Nonlinear features of internal waves off Baja California as observed from the SEASAT imaging radar. Journal of Geophysical Research, 88(C7), 4459-4466. https://doi.org/10.1029/JC088ic07p04459

Ardhuin, F., Aksenov, Y., Benetazzo, A., Bertino, L., Brandt, P., Caubet, E., et al. (2018). Measuring currents, ice drift, and waves from space: The sea surface kinematics multiscale monitoring (skim) concept. Ocean Science, 14(3), 337-354.

Ardhuin, F., Chapron, B., \& Collard, F. (2009). Observation of swell dissipation across oceans. Geophysical Research Letters, 36, L06607. https://doi.org/10.1029/2008GL037030

Ardhuin, F., Collard, F., Chapron, B., Girard-Ardhuin, F., Guitton, G., Mouche, A., \& Stopa, J. E. (2015). Estimates of ocean wave heights and attenuation in sea ice using the SAR wave mode on Sentinel-1a. Geophysical Research Letters, 42, 2317-2325. https://doi.org/10.1002/ 2014GL062940 
Ardhuin, F., Stopa, J., Chapron, B., Collard, F., Smith, M., Thomson, J., et al. (2017). Measuring ocean waves in sea ice using SAR imagery: A quasi-deterministic approach evaluated with Sentinel-1 and in situ data. Remote Sensing of Environment, 189(Supplement C), 211-222

Beal, R. C., Tilley, D. G., \& Monaldo, F. M. (1983). Large-and small-scale spatial evolution of digitally processed ocean wave spectra from SEASAT synthetic aperture radar. Journal of Geophysical Research, 88(C3), 1761-1778. https://doi.org/10.1029/ JC088iC03p01761\%

Chapron, B., Collard, F., \& Ardhuin, F. (2005). Direct measurements of ocean surface velocity from space: Interpretation and validation Journal of Geophysical Research, 110, C07008. https://doi.org/10.1029/2004JC002809

Chapron, B., Johnsen, H., \& Garello, R. (2001). Wave and wind retrieval from SAR images of the ocean. Annales Des Télécommunications, 56(11), 682-699. https://doi.org/10.1007/BF02995562

Collard, F., Ardhuin, F., \& Chapron, B. (2009). Monitoring and analysis of ocean swell fields from space: New methods for routine observations. Journal of Geophysical Research, 114, C07023. https://doi.org/10.1029/2008JC005215

Engen, G., \& Johnsen, H. (1995). Sar-ocean wave inversion using image cross spectra. IEEE Transactions on Geoscience and Remote Sensing, 33(4), 1047-1056.

Furevik, B. R., Schyberg, H., Noer, G., Tveter, F., \& Rhrs, J. (2015). ASAR and ASCAT in polar low situations. Journal of Atmospheric and Oceanic Technology, 32(4), 783-792. https://doi.org/10.1175/JTECH-D-14-00154.1

Jackson, C. R., \& Apel, J. R. (2004). Synthetic aperture radar marine user's manual. U.S. DEPARTMENT OF COMMERCE National Oceanic and Atmospheric Administration National Environmental Satellite, Data, and Information Service Office of Research and Applications.

Johannessen, J. A., Chapron, B., Collard, F., Kudryavtsev, V., Mouche, A., Akimov, D., \& Dagestad, K.-F. (2008). Direct ocean surface velocity measurements from space: Improved quantitative interpretation of Envisat ASAR observations. Geophysical Research Letters, 35, L22608. https://doi.org/10.1029/2008GL035709

Johannessen, J. A., Shuchman, R. A., Johannessen, O. M., Davidson, K. L., \& Lyzenga, D. R. (1991). Synthetic aperture radar imaging of upper ocean circulation features and wind fronts. Journal of Geophysical Research, 96(C6), 10,411-10,422. https://doi.org/10.1029/ 91JC00301

Johnsen, H., \& Collard, F. (2009). Sentinel-1 ocean swell wave spectra (OSW) algorithm definition. S1-TN-NRT-52-7450 Tromso, Norway: Norut.

Juszko, B.-A., Marsden, R. F., \& Waddell, S. R. (1995). Wind stress from wave slopes using Phillips equilibrium theory. Journal of Physical Oceanography, 25(2), 185-203. https://doi.org/10.1175/1520-0485(1995)025<0185:WSFWSU>2.0.CO;2

Kerbaol, V., Chapron, B., \& Vachon, P. W. (1998). Analysis of ERS-1/2 synthetic aperture radar wave mode imagettes. Journal of Geophysical Research, 103(C4), 7833-7846. https://doi.org/10.1029/97JC01579

Kudryavtsev, V., Kozlov, I., Chapron, B., \& Johannessen, J. A. (2014). Quad-polarization SAR features of ocean currents. Journal of Geophysical Research: Oceans, 119, 6046-6065. https://doi.org/10.1002/2014JC010173

Li, H., Mouche, A., Stopa, J. E., \& Chapron, B. (2018). Calibration of the normalized radar cross section for Sentinel-1 wave mode. IEEE Transactions on Geoscience and Remote Sensing, 99, 1-9. https://doi.org/10.1109\%2Ftgrs.2018.2867035

Lyzenga, D. R. (1986). Numerical simulation of synthetic aperture radar image spectra for ocean waves. IEEE Transactions on Geoscience and Remote Sensing, GE-24(6), 863-872.

Montgomery, M. T., \& Farrell, B. F. (1992). Polar low dynamics. Journal of the Atmospheric Sciences, 49(24), 2484-2505. https://doi.org/10.1175/1520-0469(1992)049<2484:PLD>2.0.CO;2

Mouche, A. A., Collard, F., Chapron, B., Dagestad, K. F., Guitton, G., Johannessen, J. A., et al. (2012). On the use of Doppler shift for sea surface wind retrieval from SAR. IEEE Transactions on Geoscience and Remote Sensing, 50(7), 2901-2909.

Nilsen, V., Engen, G., \& Johnsen, H. (2018). A novel approach to SAR ocean wind retrieval. Presentation on SeaSAR 2018, ESA-ESRIN, Frascati (Rome), Italy, 2018, url=http://seasar2018.esa.int/files/presentation145.pdf.

Nouguier, F., Chapron, B., Collard, F., Mouche, A. A., Rascle, N., Ardhuin, F., \& Wu, X. (2018). Sea surface kinematics from near-nadir radar measurements. IEEE Transactions on Geoscience and Remote Sensing, 56(10), 6169-6179.

Quilfen, Y., Chapron, B., Bentamy, A., Gourrion, J., El Fouhaily, T., \& Vandemark, D. (1999). Global ERS 1 and 2 and NSCAT observations: Upwind/crosswind and upwind/downwind measurements. Journal of Geophysical Research, 104(C5), 11,459-11,469. https://doi.org/10.1029/1998JC900113

Rouault, M. J., Mouche, A., Collard, F., Johannessen, J. A., \& Chapron, B. (2010). Mapping the Agulhas Current from space: An assessment of ASAR surface current velocities. Journal of Geophysical Research, 115, C10026. https://doi.org/10.1029/2009JC006050

Smirnova, J. E., Golubkin, P. A., Bobylev, L. P., Zabolotskikh, E. V., \& Chapron, B. (2015). Polar low climatology over the nordic and Barents Seas based on satellite passive microwave data. Geophysical Research Letters, 42, 5603-5609. https://doi.org/10.1002/2015GL063865

Stopa, J. E., Ardhuin, F., Chapron, B., \& Collard, F. (2015). Estimating wave orbital velocity through the azimuth cutoff from space-borne satellites. Journal of Geophysical Research: Oceans, 120, 7616-7634. https://doi.org/10.1002/2015JC011275

Stopa, J. E., \& Mouche, A. (2017). Significant wave heights from Sentinel-1 SAR: Validation and applications. Journal of Geophysical Research: Oceans, 122, 1827-1848. https://doi.org/10.1002/2016JC012364

Stopa, J. E., Sutherland, P., \& Ardhuin, F. (2018). Strong and highly variable push of ocean waves on Southern Ocean sea ice. Proceedings of the National Academy of Sciences, 115(23), 5861-5865.

Torres, R., Snoeij, P., Geudtner, D., Bibby, D., Davidson, M., Attema, E., et al. (2012). GMES Sentinel-1 mission. Remote Sensing of Environment, 120(Supplement C), 9-24. The Sentinel Missions - New Opportunities for Science. 